Bibliotecas. Vol 36, № 2, enero - junio, 2018. EISSN: 1659-3286

URL: http://www.revistas.una.ac.cr/index.php/bibliotecas/index

DOI: http://dx.doi.org/10.15359/rb.36-2.1

Licencia: Creative Commons (BY-NC-SA) 4.0 Internacional

\title{
Factores para la preservación digital sustentable de archivos sonoros
}

\section{Sustainable Digital Preservation Factors for Sound Archives}

\author{
Perla Olivia Rodríguez Reséndiz* \\ Instituto de Investigaciones Bibliotecológicas y de la Información \\ Universidad Nacional Autónoma de México
}

Recibido: 04 de mayo de 2017 Aceptado: 01 de diciembre de 2017

Corregido: 08 de enero 2018 Publicado: 11 de mayo de 2018

\section{Resumen}

La preservación de documentos digitales es uno de los retos más relevantes que tienen ante sí los archivos sonoros. La conservación y acceso a grandes volúmenes de información digital sonora que se resguarda en los archivos sonoros es una tarea compleja, que requiere un cambio de perspectiva en la forma tradicional de preservar los documentos sonoros. Para enfrentar este desafío, se propone la preservación digital sustentable como el procedimiento para conservar y dar acceso a largo plazo a los contenidos sonoros. La preservación digital sustentable se basa en tres factores: a) infraestructura tecnológica compartida; b) reducción del consumo de energía y del impacto ambiental y; c) continuidad y autogeneración de recursos económicos.

Palabras clave: Preservación; Preservación digital; Archivos sonoros; Sustentabilidad. 


\begin{abstract}
The preservation of digital documents is one of the most relevant challenges facing sound archives. The conservation and access to large volumes of digital sound information that is protected in sound archives is a complex task that requires a change of perspective in the traditional way of preserving sound documents. To face this challenge, it is proposed the sustainable digital preservation as the procedure to conserve and give access to the long-term sound content. The digital sustainable preservation takes in consideration three factors: a) shared technology infrastructure, b) reduce the consumption energy and the impact environmental and c) continuity and self-generation of economic resources.
\end{abstract}

Keywords: Preservation; Digital Preservation; Sound Archives, Sustainable.

\title{
I. Introducción
}

Las grabaciones sonoras, producidas durante más de un siglo y medio en una amplia gama de soportes y formatos, son documentos de valor histórico, social y cultural. Procurar su salvaguarda como patrimonio inmaterial es una tarea ineludible (Unesco, 1980). Desde 1860, fue posible fijar el sonido en una amplia gama de materiales analógicos y digitales. Los soportes analógicos en que el sonido ha sido grabado pueden clasificarse en mecánicos 0 bien magnéticos. Son soportes mecánicos los rollos de papel de pianolas; los cilindros (cera, celuloide y amberol); los discos de surco grueso (78 rpm y similares); los discos de transcripción (prensado) y los discos de lacado instantáneo. Los soportes analógicos magnéticos son los carretes de alambre, las cintas de carrete abierto, los casetes y los cartuchos. Los soportes digitales son los discos ópticos (minidisco, disco compacto -CD-; el disco versátil -DVD- y el Blu Ray), las cintas digitales (Digital Audio Tape -DAT- y Linear Tape Open -LTO- y los discos duros). 
La digitalización es el único método, conocido hasta ahora, para que los audios fijados en soportes analógicos se preserven a largo plazo. Para llevar a cabo esta tarea se han puesto en marcha sendos proyectos para transferir contenidos grabados en soportes analógicos a plataformas digitales. La digitalización ha sido una de las tareas más relevantes de los archivos sonoros en los últimos años.

El incremento de contenidos digitales provenientes de la digitalización; así como la creciente producción de materiales cuyo origen es digital, evidenció que los métodos, técnicas y tecnologías empleados en la preservación de soportes analógicos son insuficientes frente al dominio de los soportes digitales. Fue necesario considerar otro método para conservar y dar acceso a las nacientes colecciones digitales sonoras: la preservación digital.

La preservación digital se basa en el almacenamiento de contenidos en soportes digitales que están expuestos a una amplia gama de riesgos que pueden provocar pérdida de datos. Además, ningún soporte de almacenamiento digital es permanente y el tiempo de vida útil, antes de que sea obsoleto es menor al de los soportes analógicos. Situaciones a las que se suma la falta de continuidad en la asignación de recursos económicos para mantener los sistemas de gestión y almacenamiento masivo digital; el cambio en la dinámica organizacional del archivo sonoro debido a la transición de la preservación analógica a la digital; así como el impacto que tiene la tecnología utilizada en la preservación digital en el medio ambiente. Bajo estas consideraciones, la permanencia de la información digital de los archivos sonoros a largo plazo es un problema de interés contemporáneo que no ha sido resuelto.

\section{Objetivo}

La necesidad de preservar grandes volúmenes de contenidos sonoros digitales, para que sean escuchados por las generaciones del futuro, ha dado lugar a la formulación de una serie de interrogantes y preocupaciones en torno a cómo garantizar la persistencia de los contenidos digitales sonoros en el tiempo. 
La preservación digital no es una acción que inicia y concluye en un determinado periodo. La preservación digital es una tarea permanente que se desarrolla a lo largo del tiempo y es, además, un ámbito complejo en el que intervienen diversos factores.

En consecuencia, se propone a la preservación digital sustentable como el medio para que los contenidos permanezcan a lo largo del tiempo. Bajo esta consideración, el objetivo de este artículo es identificar, analizar y formular los principales factores que intervienen en la preservación digital sustentable fin de garantizar la conservación y el acceso a las colecciones sonoras para el futuro.

\section{Metodología}

Este artículo es resultado de la lectura y análisis de artículos de investigación, informes y estudios recientes en torno a la preservación digital de archivos sonoros. La etapa de revisión documental ha sido fundamental para establecer un panorama contemporáneo en torno a la situación actual y problemas de la preservación digital en general y de los archivos sonoros en particular. Por otra parte, este artículo también se ha nutrido de la observación y análisis de caso en archivos sonoros de fonotecas de alcance nacional, emisoras de radio y centros de investigación. Así, desde la perspectiva profesional se han podido incorporar observaciones que dan cuenta de la situación actual que enfrentan las instituciones de la memoria sonora y de la apremiante situación que existe en relación con la preservación de las colecciones digitales sonoras.

La estructura del artículo inicia con una revisión de las aportaciones y discusiones científicas y políticas en relación con el término sustentable. Después, se define qué es la preservación digital sustentable. Más adelante, se establecen los factores: infraestructura tecnológica compartida; reducción del consumo de energía y el impacto ambiental, así como continuidad y autogeneración de recursos. Cada uno de estos factores se desarrolla de manera independiente. 


\section{Sustentabilidad y preservación digital}

El incremento de información ha ocasionado problemas en archivos y bibliotecas que sin haberlo considerado deben conservar y gestionar grandes volúmenes de información digital. La tendencia de crecimiento de las colecciones digitales contrasta con la carencia de planes y documentos de preservación digital a largo plazo. Solo el $26.1 \%$ de las instituciones europeas que resguardan colecciones digitales posee un plan de preservación digital que describe el proceso de organización para la preservación de los documentos digitalizados y de aquellos cuyo origen es digital; y el $48 \%$ aún no tiene una solución de preservación de largo plazo (Stroeker y Panteia, 2012). Esta situación avizora que la preservación digital a largo plazo es un problema social, un ámbito de interés y preocupación internacional, debido a que la información digital es frágil y está expuesta a la pérdida y degradación (Blue Ribbon, 2008; UNESCO, 20003). En consecuencia, es necesario modificar la forma en que se preservan las colecciones digitales, por una perspectiva de largo plazo, que persista a lo largo del tiempo. Lo que significa que, si deseamos que las colecciones permanezcan en el futuro, la gestión sustentable debe ser un cambio estratégico en el siglo XXI (Unión Europea, 2014).

Para denominar el carácter de permanencia de los contenidos digitales sonoros (audio y metadata) en el tiempo, se utilizará el término sustentable, que es aquello que "se puede sustentar o defender con razones" (DRAE, 2014). La palabra sustentable es sinónimo de sostenible y es un proceso "que puede mantenerse por sí mismo" (DRAE, 2014: s/p). En inglés, sustentable y sostenible se traducen con el término sustainable.

El término sustentable se ha utilizado en discusiones políticas y científicas en las últimas cuatro décadas. En los años 1960, se utilizó con un sentido económico (Bradley, 2007a); en la década de 1970 se vinculó a la necesidad de cuidado del medio ambiente y en los años 1980 se diseminó y popularizó gracias al Informe Brundtland (1987) que utilizó por primera vez el término desarrollo sostenible. 
En los primeros años del siglo XXI, la generación, distribución y conservación de una gran cantidad de recursos de información, a través de las tecnologías de información y comunicación, motivó que el término sustentable se asociara a la permanencia de la información digital. Se llevaron a cabo investigaciones que vinculan el concepto desarrollo sustentable -sustainable, en inglés- a términos como sociedad de la información y economía del conocimiento; y se formuló el concepto de información sustentable (Nolin, 2010). Además, Chowdhury (2012) diseñó el modelo de sistemas y servicios de información digital sustentable. Las contribuciones científicas se han centrado en cómo hacer que la información digital permanezca a largo plazo.

En el ámbito de los archivos sonoros se carece de aportaciones en torno a lo que es la preservación digital sustentable. La contribución más cercana fue formulada por Kevin Bradley (2007a) quien advirtió que la preservación digital es una parte significativa e integral de la sustentabilidad digital. La sustentabilidad digital es el contexto organizacional, socio-técnico y económico en el que se desarrolla la preservación digital (Bradley, 2007a). Esta aportación, formulada cuando iniciaron los primeros proyectos de digitalización de colecciones sonoras, no sólo se centra en la tecnología y en la secuencia de datos que se puedan mantener por sí mismos; establece "claramente que no es posible preservar información digital sin infraestructura organizacional, económica, social y técnica sustentable, ni es sensato preservar material sin valor sostenido" (Bradley, 2007a, p. 157).

\section{Factores para la preservación digital sustentable de archivos sonoros}

La preservación digital ha sido definida como una tarea global, continua y compleja en la que han de tenerse en cuenta factores físicos y lógicos de la información (Romero, 2006); cuyo fin ulterior y a largo plazo asegura la permanencia y acceso del contenido (Voutssas, 2009), a partir de políticas de conservación y seguridad informática que aseguran su mantenimiento y uso a largo plazo (Termens, 2013). 
La preservación digital es una tarea permanente, que se desarrolla a lo largo del tiempo, a través de la cual se asegura la conservación y acceso a los contenidos. Bajo esta perspectiva, la preservación digital de archivos sonoros puede ser entendida como el método sustentable a través del cual se conservan, administran, gestionan y se proporciona acceso, difusión y reaprovechamiento permanente -por siempre- al audio digital, también denominado esencia o media y a los metadatos (Rodríguez, 2016a). Desde esta perspectiva, lo sustentable es la meta a alcanzar para que a través de la preservación digital permanezca la información digital a través del tiempo. Para alcanzar esta meta se propone un conjunto de factores a tomar en consideración en la tarea permanente de preservación digital. Los factores a considerar son:

- Infraestructura tecnológica compartida.

- Reducción del consumo de energía y el impacto ambiental.

- Continuidad y autogeneración de recursos económicos.

\section{a. Infraestructura tecnológica compartida}

Los primeros pasos en la preservación de contenidos digitales de los archivos sonoros se basaron en la adquisición de soluciones tecnológicas propietarias diseñadas a medida del archivo. La falta de continuidad, en el suministro de recursos financieros y en la actualización tecnológica, es la más grande amenaza social, económica y política que afrontan los archivos sonoros (Rodríguez, 2016b) y que pone en riesgo de pérdida las colecciones digitales sonoras.

Además, el incremento y la acumulación de contenidos digitales en los archivos sonoros evidencian que la capacidad de almacenamiento ha superado las necesidades convencionales (Bradley, 2007b) y que los soportes de almacenamiento de datos están "en constante progreso y desarrollo, lo que representa cambios inevitables y peligro de obsolescencia continua" (IASA, 2006: 60). Por ejemplo, las cintas para almacenamiento de datos ofrecen una nueva generación, aproximadamente cada 18 meses o dos años; muchos formatos son compatibles al menos con la generación anterior (IASA, 2006). Hasta ahora, no 
existe una tecnología que dure para siempre y cuyo almacenamiento de datos sea seguro. Por ello, para algunos archivos que ya tienen colecciones digitales sonoras, la migración y la emulación, han sido en los últimos años ámbitos de interés como métodos de preservación digital.

En la actualidad son escasas las investigaciones encaminadas a crear soportes de almacenamiento que sean perdurables. El interés de la comunidad científica por encontrar un soporte único y permanente de almacenamiento masivo digital ha sido sustituido por investigar las arquitecturas de sistemas, estándares, metadatos y herramientas (Bradley, 2007a).

Por otra parte, el archivo digital sonoro experimenta cambios debido a la aparición de modelos arquitectónicos emergentes de tecnologías de la información en los que se ofrecen el software y la infraestructura como servicios. La visión monolítica del software ha comenzado a ser suplantada por servicios de flujos de trabajo externos para la preservación debido a que "la inversión en sistemas únicos para la preservación digital contribuye a la falta de interoperabilidad, a la fragmentación de los recursos y a la creación de silos digitales" (Fresa, Justrell y Prandoni, 2015). Este tipo de arquitecturas no se pueden sostener en el largo plazo desde una perspectiva sustentable. En cambio "las soluciones compartidas para la creación, almacenamiento y uso de recursos digitales, incluyendo las e-infraestructuras, se convertirán en el mayor componente de la economía del conocimiento futuro" (Fresa, Justrell y Prandoni, 2015). Por lo tanto, la perspectiva de preservación digital sustentable ha de tomar en consideración la infraestructura compartida para instituciones de la memoria sonora.

Las soluciones compartidas entre archivos podrían considerar la participación de Universidades y Centros de Investigación a fin de incentivar la investigación aplicada en torno al archivo digital y el uso de tecnologías innovadoras, como e-infraestructuras, e-servicios, la nube y los micros servicios, así como de tecnologías que no han sido adoptadas en la preservación digital como el Big Data (Ruusalepp y Dovreba, 2013). Sumado a lo cual, se debe asegurar que las soluciones tecnológicas consideren la interoperabilidad y acceso actual 
y futuro. Entre más se pruebe que un documento digital sonoro se puede escuchar en diversas plataformas, se garantiza su acceso y con ello, un aspecto clave de la preservación digital (Bradley, 2007a).

\section{b. Reducción del consumo de energía y el impacto ambiental}

El crecimiento de contenidos digitales (media y metadatos) en los archivos sonoros requiere del uso permanente de energía. Los SGAM ampliamente definidos por la IASA (2005), requieren para su operación y mantenimiento de un sistema de energía ininterrumpida durante 24 horas, los 365 días del año. La falta de continuidad en el abasto de energía eléctrica puede provocar la pérdida de datos.

EI SGAM puede ser definido como un centro de datos; es decir, como el repositorio centralizado, ya sea físico o virtual, para el almacenamiento, gestión y difusión de datos de información organizada alrededor de un cuerpo determinado de conocimiento o perteneciente a un negocio particular (Tech Target, 2012). Solo en 2011 se reportaron 509147 centros de datos en el mundo (Miller, 2011). El crecimiento de los centros de datos se relaciona con el consumo de energía y el impacto ambiental. Se ha advertido que las tecnologías de la información y la comunicación generan el $2 \%$ de las emisiones de $\mathrm{CO} 2$, tanto como el transporte aéreo (The Climate Group, 2008). Se prevé que esta cifra se duplique en 2020. En consecuencia, la conservación, gestión y acceso a grandes cantidades de información digital, a través de los centros de datos es un tema de relevancia contemporánea. Las empresas que trabajan con grandes volúmenes de información han comenzado a evaluar su impacto ambiental; de hecho, los centros de datos de Reino Unido vinculan el término sustentable con gasto de energía, cuidado del agua y generación de basura (Jones, P. y otros, 2013).

En relación con el consumo de energía y el impacto ambiental el investigador Wissner-Gross de la Universidad de Harvard, señaló que una búsqueda estándar en Google produce 7 gramos de $\mathrm{CO} 2$, debido a que requiere de energía eléctrica para conectar varias bases de 
datos (BBC, 2009). Con un billón de búsquedas en Google que se realizan todos los días, hay un billón de gramos de $\mathrm{CO} 2$ que son emitidos a la atmósfera.

Chowdhury (2012) ha estudiado cómo el sector de servicios de la información hace uso de tecnologías de información y comunicación (TIC), cuyos equipos generan una cantidad significativa de gas de efecto invernadero. Los centros de datos son los principales usuarios de energía (Jones, P. y otros, 2013).

El gasto de energía y el impacto ambiental que ocasiona la preservación digital de archivos sonoros es un tema poco explorado. Solo se cuenta con las evidencias presentadas por Tadic (2015) durante la Conferencia Anual de la IASA en París, cuando llamó a reflexionar en relación con la energía necesaria para mantener, con una copia espejo, de forma permanente 29.2 exabytes de datos, que corresponden a 400000000 horas de contenidos sonoros y audiovisuales durante 24 horas los 365 días al año. De estos, 250 millones de horas son de audio y equivalen a 500 petabites. Otro aspecto señalado por Tadic (2015) fue la producción de chatarra tecnológica, tanto de los soportes analógicos, como de la tecnología digital que sistemáticamente queda en desuso. La basura tecnológica se suma a lista de elementos tóxicos que dañan la salud y el medio ambiente (Vilches, A; Macías, O y Pérez, D.2014). Situación que afecta a los archivos sonoros porque los equipos de grabación y reproducción sonora, derivado de la obsolescencia tecnológica, quedan en desuso de forma cíclica.

El gasto de energía y el impacto ambiental que ocasiona la preservación digital de los archivos sonoros son temas que deben ser analizados en foros de alcance internacional; por ejemplo, en la Convención del Cambio Climático (ONU, 2015) que llevó a suscribir el Acuerdo de París. Los archivos sonoros deben incorporar estrategias sustentables a sus amplias y complejas tareas de preservación. Es probable que sigan los pasos de los centros de datos que proyectan el uso de energías renovables, especialmente la solar; la incorporación de la nube como medio almacenamiento y la reutilización de tecnologías, entre otras estrategias (Chowdhury, 2012 y Emerson Network, 2014), para que dentro de una década el gasto de energía y el impacto ambiental disminuyan. 


\section{c. Continuidad y autogeneración de recursos económicos}

El alto costo en la instalación de tecnología para la preservación digital evidenció la confrontación entre las tradicionales estrategias económicas de preservación y cómo hacer que la preservación digital sea una actividad económica sustentable (Lavoie, 2004) y puso de manifiesto que el mayor riesgo de tener un acceso sostenido es el económico (Bradley, 2007a). Muchas colecciones analógicas se mantuvieron durante varios años y décadas con pocos o nulos recursos económicos. Ante el abandono de las colecciones solo el trabajo incansable y comprometido de los archivistas las protegió de la destrucción. Pero en el caso de las colecciones digitales la negligencia benigna es catastrófica (Walters y Skinner, 2009).

Los contenidos digitales son altamente vulnerables porque el almacenamiento digital no es estable y la preservación es un ámbito relativamente nuevo y poco estudiado. Según Walters y Skinner (2009) "la infraestructura digital con la que los activos digitales existen cambia siempre en términos de hadware y software, protocolos y estándares" (p. 259) en un corto periodo.

Muchas iniciativas están en los primeros pasos y no cuentan con estimaciones de los costos totales para mantener los materiales digitales por un largo periodo (Lavoie, 2003). Se centran en obtener recursos para la digitalización y olvidan que la obsolescencia de la tecnología digital es también un problema económico. Lo cierto es que obtener financiamiento solo una vez, es una estrategia inadecuada para la preservación de los objetos digitales. Las instituciones que conceden recursos económicos no pueden pensar que si otorgan dinero para dos o tres años garantizan la preservación a largo plazo. La permanencia de los contenidos digitales requiere de una perspectiva económica sustentable. La preservación digital es una actividad que se lleva a cabo en el tiempo, es decir, no se puede decir que un documento esté preservado (Edmodson, 2008).

Los documentos digitales sonoros son bienes intangibles culturales y no generan beneficios económicos a través de los cuales las instituciones de la memoria y la sociedad pueden 
beneficiarse. Por ello, en ocasiones la preservación de este tipo de documentos se relega; sin embargo, como han apuntado Walters y Skinner (2009) una de las mejores defensas ante esta situación es considerar que estamos ante un futuro digital en el que la propiedad y la administración del contenido cultural se llevarán a cabo sobre todo dentro de un medio digital. $\mathrm{Si}$ las instituciones que asignan los recursos, deciden no preservar, deben tener en cuenta los costos culturales, políticos, científicos e institucionales que trae consigo esta decisión (Walters y Skinner, 2009). Las instituciones que cuiden sus contenidos digitales, digitalicen sus colecciones, inviertan en infraestructura digital, capaciten a su personal para manejar contenidos digitales son las instituciones del futuro.

Por su parte, los responsables de un archivo sonoro necesitan formular planes de presupuesto a largo plazo, considerando que la preservación digital debe basarse en el menor costo durante todo el ciclo de vida del documento digital y no solamente durante la digitalización (Lavoie, 2003). Para el diseño de un plan de presupuesto a largo plazo, los archivos deben identificar tanto las actividades clave de la preservación digital; las tareas que se deben eliminar o transformar para garantizar la operación del archivo digital; los servicios y tecnologías que se pueden compartir con otras instituciones para aminorar costos de operación; los servicios de información actuales y futuros; los servicios que tendrán costo y las estrategias para recaudación de fondos (Blue Ribbon, 2008).

De forma paralela al proceso de procuración de recursos económicos para el archivo, este debe formular propuestas innovadoras y creativas de acceso, uso y reaprovechamiento que pongan en valor los contenidos. El acceso a los contenidos digitalizados crea valor (Arquero, 2014), para las instituciones de la memoria, las industrias creativas, las instituciones culturales y el sector educativo. El reaprovechamiento educativo y cultural del patrimonio digital sonoro forma parte del ecosistema digital contemporáneo. La forma de preservar un documento digital en el presente determinará y potenciará el valor de uso que pueda tener en el futuro. El valor de uso de un documento sonoro, una vez que ha sido digitalizado, es el concepto sobre el que se deben basar las actividades de preservación digital sustentable. 


\section{Conclusiones}

Para garantizar la permanencia de los contenidos sonoros que han sido digitalizados, o bien cuyo origen es digital, es necesario un cambio de perspectiva sobre la preservación tradicional de documentos analógicos a la digital. La preservación digital debe ser una tarea continua a través de la cual se almacenen grandes volúmenes de información digital.

El mayor reto que enfrentan las instituciones de la memoria sonora es procurar que los documentos digitales permanezcan. Para denominar el carácter de permanencia en el tiempo se ha propuesto la preservación digital sustentable como el proceso a través del cual es posible conservar y dar acceso a largo plazo a las colecciones digitales de los archivos sonoros.

La preservación digital sustentable en un archivo sonoro se basa en tres factores: infraestructura tecnológica compartida; reducción del consumo de energía y del impacto ambiental y continuidad y autogeneración de recursos económicos. Cada uno de estos factores puede ser entendido como una línea maestra en el archivo sonoro y ser incorporado como parte de los planes y programas de preservación digital a largo plazo. Los factores propuestos pretenden ser una herramienta conceptual para comprender el complejo desafío que significa la preservación digital sustentable de los archivos sonoros.

\section{Referencias}

Arquero, R. y Cobo, S. (2014). "Modelo de negocio y visión estratégica de Europeana". En Ramos, L.; Arquero, R. Europeana. La plataforma del patrimonio cultural europeo. Ediciones TREA. España.

BBC Mundo. (2009). El costo ambiental de "googlear". BBC. Disponible en http://news.bbc.co.uk/hi/spanish/science/newsid 7823000/7823918.stm

Blue Ribbon. (2008). The Blue Ribbon Task Force on Sustainable Digital Preservation and Access. Interim Report. Disponible en http://brtf.sdsc.edu/biblio/BRTF Interim Report.pdf 
Bradley, K. (2007a). Defining digital sustainability. Library Trends, Volume 56 (1). Disponible en http://muse.jhu.edu/journals/library trends/toc/lib56.1.html

Bradley, K. (2007b). Hacia un sistema de almacenamiento y preservación en código abierto. Subcomité de tecnología del Programa Memoria del Mundo de la Unesco. Traducción al español Ing. Antonio Limas. CONACULTA, México.

Climate Group. (2008). SMART2020: enabling the low carbon economy in the information age. Disponible en www.theclimategroup.org/publications/2008/6/19/smart2020-enabling- thelow-carbon-economy-in-the-information-age/

Chowdhury, G. (2012). Sustainability of digital information services, en Journal of Documentation, Vol. 69 (5). DOI http://dx.doi.org/10.1108/JD-08-2012-0104

Diccionario de la lengua española (2014). Última edición es la 23. a Disponible en http://lema.rae.es/drae/?val=sustentable

Edmondson, R. (2008). Filosofía y principios de los archivos audiovisuales. UNESCO.

Emerson Network. (2014). Data center 2025: exploring the possibilities, en Emerson Network Power. Disponible en http://www.emersonnetworkpower.com/en-US/LatestThinking/Data-Center-2025/Pages/Data-Center-2025-Report.aspx

Fresa, A.; Justrell, B.; Prandoni, C. (2015). Digital curation and quality standards for memory institutions: PREFORMA research Project. Archival Science. DOI 10.1007/s10502-015$\underline{9242-8}$

IASA. (2005). IASA-TC 03 La salvaguarda del patrimonio sonoro: Ética, principios y estrategia de preservación. Versión traducida al español. México: IASA Radio Educación.

IASA. (2006). Lineamientos para la producción y preservación de objetos de audio digitales. TC-04. Asociación Internacional de Archivos Sonoros y Audiovisuales (IASA). UNESCO.

Informe Brundtland. (1987). Disponible en: http://www.un.org/es/comun/docs/?symbol=A/42/427

Jones, P.; Hillier, D.; Comfort, D.; Clarke-Hill, C. (2013) "Towards sustainable data centre operations in the UK", en Journal of Property Investment \& Finance, 2013. DOI: $\underline{10.1108 / 1463578131129299}$ 
Lavoie, B. (2003). The Incentives to Preserve Digital Materials: Roles, Scenarios, and Economic Decision-Making. Reproduction, 45. Disponible en: http://www.oclc.org/research/projects/digipres/incentives-dp.pdf

Lavoie, B. (2004)."Of Mice and Memory: Economically Sustainable Preservation for the Twenty-first Century," en Access in the Future Tense. Washington, DC: Council on Library and Information Resources. Disponible en: http://www.clir.org/pubs/reports/pub126/lavoie.html

Miller, R. (2011).“How many Data Centers? Emerson says 500,000”. Data Center Knowledge. Disponible en: http://www.datacenterknowledge.com/archives/2011/12/14/how-many-datacenters-emerson-says-500000/

Nolin, J. (2010). Sustainable information and information science. Information Research-an International Electronic Journal.

Organización de Naciones Unidas (ONU). (2015). Convención Marco de Naciones Unidas sobre el Cambio Climático. Disponible en https://unfccc.int/resource/docs/2015/cop21/spa/l09s.pdf

Rodríguez, P. (2016a). La preservación digital sustentable de archivos sonoros. México: Instituto de Investigaciones Bibliotecológicas y de la Información (UNAM).

Rodríguez, P. (2016b). La preservación digital sonora. Investigación Bibliotecológica: Archivonomía, Bibliotecología e Información, 68.

Romero, J. (2006). El papel de los metadatos en la preservación digital. El profesional de la información, 15 (2).

Ruusalepp, R. y Dobreva, M. (2013). Digital Preservation Services: State of the Art Analysis. European Commission, FP7. Disponible en: https://www.um.edu.mt/library/oar/bitstream/handle/123456789/311/dobreva Preservati on Services study.pdf? sequence $=1$ \&isAllowed $=y$

Stroeker, N.; Panteia, R. (2012). Survey Report on Digitisation in European Cultural Heritage Institutions. EUMERATE. ICT Policy Support Programme part of the Competitiveness and Innovation Framework Programme. 
Tadic, L. (2015). The enviromental impact in digital preservation. Ponencia presentada en AMIA Conference, Portland, Estados Unidos.

TechTarget. (2012). Data center definition. Tech Target. Disponible en: http://searchdatacenter.techtarget.com/definition/data-center

Unión Europea. (2014). Conclusions on digital heritage as a strategic resource for a sustainable Europa. Education, Youth, Culture and Sport Council meeting Brussels.

UNESCO (1980). Recomendación sobre la Salvaguardia y la Conservación de las Imágenes en Movimiento: UNESCO. Disponible en: http://portal.unesco.org/es/ev.php

UNESCO (2003). Directrices para la preservación del patrimonio digital. UNESCO.

Térmens, M. (2013). Preservación digital. Editorial UOC. Barcelona, España.

Vilches, A; Macías, O. y Pérez, D. (2014). La transición a la sostenibilidad: un deafío urgente para la ciencia, la educación y la acción ciudadana. Organización de Estados Iberoamericanos.

Voutssás, J. (2009). Preservación del patrimonio documental digital en México. México. UNAM, Centro Universitario de Investigaciones Bibliotecológicas.

Walters, T., y Skinner, K. (2009). Economics, sustainability, and the cooperative model in digital preservation. Library Trends, 57(3). DOI: http://doi.org/10.1108/07378831011047668

\section{Notas de la Autora}

* Perla Olivia Rodríguez Reséndiz. Instituto de Investigaciones Bibliotecológicas y de la Información, de la Universidad Nacional Autónoma de México. Correo electrónico: perlaolivia@gmail.com 